\title{
Unconventional Vaccines: Progress and Challenges
}

\section{Nikolai Petrovsky ${ }^{1,2 *}$}

${ }^{1}$ Vaxine Pty Ltd, Flinders Medical Centre, Bedford Park, Adelaide, South Australia

${ }^{2}$ Flinders Medical Centre and Flinders University, Bedford Park, Adelaide, South Australia

\begin{abstract}
When vaccines are mentioned most people think of immunization against childhood infectious diseases. However, in recent years the uses to which vaccines are being put has dramatically expanded beyond traditional infectious disease applications. Vaccines currently in preclinical and clinical development target prevention or treatment of a wide range of non-infectious diseases including cancer, allergy, asthma, diabetes, rheumatoid arthritis, lupus, hypertension, heart disease, obesity, Alzheimer's disease, Parkison's disease and even nicotine and cocaine addiction. For the most part such vaccines aim to induce neutralizing antibodies against foreign or self-antigens, thereby blocking their activity and ability to induce disease. This commentary reviews key clinical advances in the area of unconventional vaccines and identifies some of the key challenges that need to be overcome in order for unconventional vaccines to move forward to medical and commercial success.
\end{abstract}

Keywords: Vaccines; Infectious diseases; Immunogenicity

\section{Introduction}

All vaccines work by the administration of a target substance called an antigen, typically a protein but also a sugar or lipid, which triggers an immune response resulting in the production of neutralizing antibodies that bind the antigen and remove it from the body. Vaccines can also activate $\mathrm{T}$ cells that then attack any cells expressing the antigen. While the immune system has primarily evolved to attack and neutralize foreign pathogens, this does not mean that it cannot respond against molecules derived from self-tissues although this can be a challenge. To overcome these problems vaccines are often formulated with adjuvants to enhance their immunogenicity. But with new vaccine applications come new challenges. One such challenge in cancer vaccines is how to break tolerance to self-antigens [1]. A challenge in allergy vaccines is how to switch an existing immune response from an $\operatorname{IgE}$ to an IgG dominant phenotype [2]. A challenge for vaccines against drug addiction is how to induce robust immune responses against nonprotein small molecules such as nicotine or cocaine [3]. Another generic challenge for all unconventional vaccines is how to maintain high levels of neutralizing antibodies in the serum over long periods of time without the need for frequent vaccine booster doses. Yet another challenge will be to demonstrate safety of each unconventional vaccine approach. Strategies must be found to overcome each of these challenges if unconventional vaccines are to become a commercial success. As will be discussed in the following sections there is a large amount of human vaccine development currently going on in this area with some positive outcomes.

\section{Vaccines Against Cancer}

In essence, cancer vaccines rely upon the induction of autoimmunity, which presents somewhat of a paradox as autoimmunity is what every infectious disease vaccine tries to avoid at all costs. Cancer vaccines typically comprise tumor-expressed antigens generally combined with an immune adjuvant designed to trigger a cellular immune response against the cancer cells. To be successful a cancer vaccine must break self-tolerance, which can be hard to achieve as normally the body tries to avoid self-reactivity. This generally requires use of a powerful inflammatory adjuvant to drive the immune system to attack tumorexpressed antigens [1]. One particular technique for administering cancer vaccines is to incubate the cancer antigen with dendritic cells in vitro thereby enabling them to uptake antigen and become activated.
The antigen-stimulated cells are then injected back into the patient. This is the basis of Sipuleucel-T vaccine (Provenge) currently the only approved human cancer vaccine [4]. Sipuleucel-T is used for treatment of prostrate cancer and is made from the patient's own lymphocytes which are incubated with a fusion protein (PA2024) consisting of two parts, the antigen prostatic acid phosphatase, an antigen present in $~ 95 \%$ of prostate cancer cells, plus granulocyte-macrophage colony stimulating factor (GM-CSF), an immune adjuvant. While Sipuleucel-T is currently the only approved therapeutic cancer vaccine out of hundreds tested in clinical trials [5], the knowledge accrued over many years of research and development make it likely that additional effective cancer vaccines will emerge over time.

\section{Vaccines Against Allergy}

Allergy immunotherapy is another promising area of unconventional vaccine development. In the United States, approximately $20 \%$ of the population or 65 million people have an allergy, with grass allergies representing $~ 50 \%$ of all allergies, animal allergies $\sim 30 \%$ and food allergies $\sim 10 \%$ [6]. Desensitization with small doses of allergens is the most effective treatment and has been practiced for almost a century, despite limited knowledge of its mechanism of action. Allergy desensitization involves subcutaneous administration of progressively higher doses of allergen, potentially lifelong. Attempts to simplify allergy desensitization to just a short course of 2-3 vaccinations have met with mixed success, which likely reflects insufficient understanding of allergy vaccine action. Potential mechanisms include IgE suppression, induction of blocking IgG antibodies and induction of regulatory $\mathrm{T}$ cells. One area of recent progress has been in application of immune adjuvants to allergy immunotherapy. Pollinex Quattro ${ }^{\oplus}$, a ragweed allergy vaccine includesthe toll-like receptor (TLR)-4 aganist monophosphoryl lipid A $[7,8]$. In an alternative approach, CYT003$\mathrm{QbG10}$, is an allergen-independent vaccine for allergic asthma and

*Corresponding author: Nikolai Petrovsky, Flinders Medical Centre and Flinders University, Bedford Park, Adelaide, South Australia, E-mail: nikolai.petrovsky@flinders.edu.au

Received May 14, 2013; Accepted May 20, 2013; Published May 24, 2013

Citation: Petrovsky N (2013) Unconventional Vaccines: Progress and Challenges. J Vaccines Vaccin 4: 186. doi:10.4172/2157-7560.1000186

Copyright: @ 2013 Petrovsky N. This is an open-access article distributed under the terms of the Creative Commons Attribution License, which permits unrestricted use, distribution, and reproduction in any medium, provided the original author and source are credited. 
rhino-conjunctivitis, which comprises a virus-like particle filled with a synthetic TLR9 agonist oligonucleotide and is postulated to work by inducing interferon alpha and ICOS ligand that stimulate regulatory $\mathrm{T}$ cells while inhibiting allergy enhancing Th2 cells [9]. Hence, new adjuvant technologies appear to be benefiting allergy immunotherapy, even while knowledge of how allergy vaccines work remains incomplete [2].

\section{Vaccines Against Chronic Degenerative Diseases}

Chronic degenerative disease is another target for unconventional vaccines. An Alzheimer's disease(AD) vaccine(AN-1792) based on the amyloid- $\beta$ peptide, $A \beta 42$, formulated with either alum or QS2 1 adjuvant successfully reversed amyloid- $\beta$ deposits and memory loss in a $\beta$ transgenic mice [10]. But in 2002 a human AN-1792 trial was halted after some patients developed meningo-encephalitis, potentially related to auto reactive T cells induced by the QS21 adjuvant [11]. Similarly, a mid-stage study of the experimental AD vaccine ACC-001 was halted after one of the patients developed CNS lesions [12]. Despite these setbacks vaccine approaches to $\mathrm{AD}$ disease have not been completely abandoned. CAD106 vaccine based on the $\mathrm{Qb}$ virus-like particle plus amyloid- $\beta$ generated antibodies against amyloid- $\beta$ in $74 \%$ of subjects [13-15]. No serious side effects were observed in the 3 year study in people aged $50-80$ years. Another AD vaccine in Phase 2 clinical trials is Affitope AD02, which comprises a pool of peptides adjuvanted with aluminium oxide [16]. Hence, despite various setbacks, progress continues to be made in development of an $\mathrm{AD}$ vaccine.

Parkinson's disease is another incurable degenerative neurological disorder that leads to movement and cognition disorders. PD01A vaccine targets alpha synuclein, mutant forms of which clump together in the brain of patients with Parkinson's disease and are thought to mediate disease pathology [17]. The PD01A vaccine comprises a pool of peptides that mimic alpha synuclein adjuvanted with aluminium oxide and has commenced clinical testing in patients with Parkinson's disease [18].

Atherosclerosis vaccines are also in development. A CETP vaccine that worked by eliciting neutralizing antibodies to cholesteryl ester transfer (CETP) raised high density lipoprotein (HDL) levels and prevented atherosclerosis in animal models. One version of the CETP vaccine, CETi-1, was a dimerized synthetic peptide, including residues 461-476 of human CETP and residues $830-843$ of tetanus toxoid [19]. The second version was PADRE-CETP a monomeric peptide containing CETPi plus the PADRE T-cell helper epitope. Both vaccines were formulated with an alum adjuvant and in some cases also included the TLR9 agonist, CPG7909. A more recent vaccine based on CETP combined with alum adjuvant, ATH03, also recently completed clinical trials [20] but the results are yet to be reported.

Type 2 diabetes and obesity are further areas of unconventional vaccine development. IL1 $\beta$ is a pro-inflammatory cytokine implicated in the pathogenes is of insulin resistance and type 2 diabetes (T2D). CYT013-IL1bQb vaccine is designed to treat T2D by induction of neutralising antibodies against interleukin(IL)1- $\beta$ [3]. Ghrelin is an endogenous peptide that enhances appetite and food intake [21]. CYT009-GhrQb vaccine was designed to reduce appetite and treat obesity by induction of neutralizing antibodies to ghrelin [3].

Hypertension is another unconventional vaccine target. Angiotensin II is an important regulator of blood pressure that causes blood vessels contraction and thereby increases blood pressure. An early anti-angiotensin vaccine that comprised angiotensin I peptides coupled to a protein hapten, was effective in reducing hypertension in a rat model but failed to demonstrate blood pressure normalization in a human trial, thought to be due to its inability to induce high titer antiangiotensin antibodies [22]. An alternative vaccine, CYT006-AngQb, where angiotensin was expressed on $\mathrm{Qb}$ viral like particles successfully induced a neutralising antibody response against angiotensin II in human subjects in a Phase 2 clinical trial but achieved only modest but significant reduction in blood pressures, indicating a need for the therapeutic potency of the vaccine to be increased $[23,24]$.

\section{Vaccines Against Autoimmune Disease}

Autoimmune diseases including multiple sclerosis (MS), rheumatoid arthritis (RA) and type 1 diabetes (T1D) are also areas of unconventional vaccine development. Tovaxin (Opexa Therapeutics) is being developed by as a personalized vaccine therapy for MS [25]. Tovaxin is manufactured using a complex method that encompasses the collection of blood from the patient, isolation of peripheral blood mononuclear cells, generation of an auto logous pool of myelin-reactive T-cells raised against selected peptides from myelin basic protein, myelin oligodendrocyte glycoprotein and proteolipid protein, and the return of these expanded irradiated $T$ cells back to the patient via subcutaneous injection. Another simpler vaccine approach for therapy of rheumatoid arthritis was based on induction of neutralizing antibodies against tumor necrosis factor (TNF)- $\alpha$, a key pro-inflammatory cytokine driving the pathogenesis of rheumatoid arthritis [26]. The problem however is that by and large such vaccines are less effective than monoclonal antibody therapeutic approaches, presumably because the antibodies induced by the vaccine are lower affinity and less durable than the monoclonal therapeutics.

In the area of T1D prevention, Diamyd, an alum-adjuvanted glutamic acid decarboxylase vaccine, showed some promise of efficacy in Phase 2 clinical testing but then failed to meet its primary efficacy endpoint (preservation of beta cell function) in a follow on Phase 3 study $[27,28]$. DiAPREV-IT, an investigator-initiated Phase II study of Diamyd in 50 children at risk of T1D is ongoing with results expected in 2015 [29]. The Diabetes Vaccine Development Centre is funding a long running study in Melbourne, Australia of nasal insulin for T1D prevention in autoantibody positive first-degree relatives [30] but with slow recruitment the study may never reach its recruitment target. Several non-antigen specific immuno-regulatory vaccines have also been shown to protect against T1D in animal models, including BCG (Bacillus Calmette-Guerin) vaccine used for TB prevention [31] and Qvax vaccine used for human $Q$ fever prevention [32]. While the BCG vaccine works via TNF- $\beta$, Qvax likely works through interferon- $\gamma$ [32], suggesting multiple immune pathways can modulate autoimmune disease induction. Nevertheless, the processes underlying autoimmune disease pathogenesis remain poorly understood, potentially helping explain the many failed attempts to use vaccines to switch off the autoimmune disease process.

\section{Vaccines Against Drug Addiction}

Drug addiction is another expanding area of unconventional vaccine research. A smoking cessation vaccine, NicVAX (Nabi Biopharmaceuticals) designed to induce anti-nicotine antibodies to block nicotine action in the brain is currently in Phase III clinical trials $[33,34]$. Nic-002, another nicotine-derived therapeutic vaccine did not achieve its primary end point of smoking cessation in Phase II clinical trials [35]. Another anti-nicotine vaccine (NicQb) comprising nicotine covalently coupled to the surface of $\mathrm{Q} \beta$ viral like particles combined with alum successfully induced anti-nicotine antibodies in human subjects [36]. 
Another drug addiction vaccine approach targets cocaine addiction. In this approach neutralizing antibodies are induced against cocaine. For example, TA-CD vaccine was shown to stimulate the generation of antibodies that bound to cocaine molecules as they entered the patient's blood stream and thereby prevented the cocaine crossing the blood brain barrier [37].

\section{Unconventional Adjuvants for Unconventional Vaccines}

Another area of critical importance to successful development of unconventional vaccines will be vaccine adjuvant technologies [38]. In most cases unconventional vaccines are based on subunit or recombinant antigen approaches, with the inherent problem of poor antigen immunogenicity. This can best be solved by addition of an appropriate adjuvant. In the case of cancer vaccines the adjuvant needs to drive a robust cellular immune response, whereas for most other unconventional vaccines the need is for an adjuvant that will drive a long-lasting serum neutralizing antibody response. Hence adjuvant selection is likely to be critical to success of many unconventional vaccines. The problem is that currently only two adjuvant platforms are approved for human use, alum-based adjuvants (aluminium hydroxide or phosphate, alone or combined with monophosphoryl lipid A) or squalene oil emulsion adjuvants (MF59, AS03), neither of which are effective in induction of strong cellular immune responses needed for maximally effective cancer vaccines and both of which suffer from potential toxicity and safety concerns [39]. More promising for unconventional vaccines may be unconventional adjuvants such as carbohydrate-based particulate vaccine adjuvants, e.g Advax ${ }^{\text {Tx }}$ delta inulin adjuvants that are well tolerated and may be better at inducing cellular immunity [40-43]. These are based on a new paradigm that suggests that inflammation may not be the best route to a robust and long-lived adaptive immune response [44]. However adjuvants alone are likely to be only part of the requirement for success of unconventional vaccines, and other requirements are to identify the rightkey antigens, and to find the best delivery routes for inducing the desired immune response.

\section{Conclusion}

The above descriptions of unconventional vaccines in clinical development are not an exhaustive listing of unconventional vaccines but rather are intended as examples of the diversity of new vaccine applications that are being explored. While to date there have been many more failures than successes with unconventional vaccine approaches this is not unusual for new areas of scientific endeavor. Notably, multiple failures were a characteristic of the early days of human monoclonal therapeutic antibodies, before sufficient lessons were learnt and technological advances made to enable monoclonal antibodies to become the success story they are today. In particular, vaccine design needs to be informed by better understanding of the pathogenic mechanism of disease, for example it is not worth investing in developing $A D$ vaccines against $A \beta$ protein, if basic science research informs us that $A \beta$ protein is not the central cause of $A D$. Hence the problem of the field is that in many cases it has got ahead of itself and antigens are being targeted that may not be critical to disease development. The problem here is that in subsequent vaccine trial failures it is impossible to know whether the failure was due to the vaccine being insufficiently potent or to the wrong antigen being targeted. There is a strong argument that for the field to advance much more basic science research needs to be done into identifying what exactly the key targets of vaccines should be for each disease indication. The success of many unconventional vaccines may also need to await the development of more effective vaccine technologies [3]. Finally, there is also much that still needs to be learnt about the science underlying the action of unconventional vaccines. But with sufficient time and effort, unconventional vaccine approaches may yet emerge as viable therapeutic strategies.

\section{References}

1. Berzofsky JA, Terabe M, Wood LV (2012) Strategies to use immune modulators in therapeutic vaccines against cancer. Semin Oncol 39: 348-357.

2. Papadopoulos NG, Agache I, Bavbek S, Bilo BM, Braido F, et al. (2012) Research needs in allergy: an EAACI position paper, in collaboration with EFA Clin Transl Allergy 2: 21

3. Bachmann MF, Jennings GT (2011) Therapeutic vaccines for chronic diseases: successes and technical challenges. Philos Trans R Soc Lond B Biol Sci 366 2815-2822.

4. Botrel TE, Clark O, Pompeo AC, Bretas FF, Sadi MV, et al. (2012) Immunotherapy with Sipuleucel-T (APC8015) in patients with metastatic castration-refractory prostate cancer (mCRPC): a systematic review and meta-analysis. Int Braz Urol 38: 717-727.

5. Ogi C, Aruga A (2013) Clinical evaluation of therapeutic cancer vaccines. Hum Vaccin Immunother 9.

6. Brusic V, Petrovsky N, Gendel SM, Millot M, Gigonzac O, et al. (2003) Computational tools for the study of allergens. Allergy 58: 1083-1092.

7. Baldrick P, Richardson D, Woroniecki SR, Lees B (2007) Pollinex Quattro Ragweed: safety evaluation of a new allergy vaccine adjuvanted with monophosphoryl lipid A (MPL) for the treatment of ragweed pollen allergy. J Appl Toxicol 27: 399-409.

8. Rosewich M, Lee D, Zielen S (2013) Pollinex Quattro: An innovative four injections immunotherapy In allergic rhinitis. Hum Vaccin Immunother 9.

9. Klimek L, Willers J, Hammann-Haenni A, Pfaar O, Stocker H, et al. (2011) Assessment of clinical efficacy of CYT003-QbG10 in patients with allergic rhinoconjunctivitis: a phase Ilb study. Clin Exp Allergy 41: 1305-1312.

10. Thatte U (2001) AN-1792 (Elan). Curr Opin Investig Drugs 2: 663-667.

11. Robinson SR, Bishop GM, Lee HG, Münch G (2004) Lessons from the AN 1792 Alzheimer vaccine: lest we forget. Neurobiol Aging 25: 609-615.

12. Ryan JM, Grundman M (2009) Anti-amyloid-beta immunotherapy in Alzheimer's disease: ACC-001 clinical trials are ongoing. J Alzheimers Dis 17: 243.

13. Kingwell K (2012) Alzheimer disease: Amyloid-î ${ }^{2}$ immunotherapy CAD106 passes first safety test in patients with Alzheimer disease. Nat Rev Neurol 8: 414

14. Winblad B, Andreasen N, Minthon L, Floesser A, Imbert G, et al. (2012) Safety, tolerability, and antibody response of active $A^{2}{ }^{2}$ immunotherapy with CAD106 in patients with Alzheimer's disease: randomised, double-blind, placebocontrolled, first-in-human study. Lancet Neurol 11: 597-604.

15. Wiessner C, Wiederhold KH, Tissot AC, Frey P, Danner S, et al. (2011) The second-generation active $A^{2}{ }^{2}$ immunotherapy CAD106 reduces amyloid accumulation in APP transgenic mice while minimizing potential side effects. J Neurosci 31: 9323-9331.

16. Schneeberger A, Mandler M, Otawa O, Zauner W, Mattner F, et al. (2009) Development of AFFITOPE vaccines for Alzheimer's disease (AD)--from concept to clinical testing. J Nutr Health Aging 13: 264-267.

17. Hutter-Saunders JA, Mosley RL, Gendelman HE (2011) Pathways towards an effective immunotherapy for Parkinson's disease. Expert Rev Neurother 11: 1703-1715.

18. Affiris AG (2013) Tolerability and Safety of Subcutaneous Administration of Two Doses of AFFITOPE ${ }^{\circledR}$ PD01A in Early Parkinson's Disease.

19. Komori T (2004) CETi-1. AVANT. Curr Opin Investig Drugs 5: 334-338.

20. Affiris AG (2012) Safety, Immunogenicity and Dose Response of ATH03, a New Vaccine Against the Cholesterol Ester Transfer Protein (CETP).

21. Allas S, Abribat T (2013) Clinical perspectives for ghrelin-derived therapeutic products. Endocr Dev 25: 157-166.

22. Brown MJ, Coltart J, Gunewardena K, Ritter JM, Auton TR, et al. (2004) Randomized double-blind placebo-controlled study of an angiotensin 
immunotherapeutic vaccine (PMD3117) in hypertensive subjects. Clin Sci (Lond) 107: 167-173.

23. Ambühl PM, Tissot AC, Fulurija A, Maurer P, Nussberger J, et al. (2007) A vaccine for hypertension based on virus-like particles: preclinical efficacy and phase I safety and immunogenicity. J Hypertens 25: 63-72.

24. Tissot AC, Maurer P, Nussberger J, Sabat R, Pfister T, et al. (2008) Effect of immunisation against angiotensin II with CYT006-AngQb on ambulatory blood pressure: a double-blind, randomised, placebo-controlled phase lla study. Lancet 371: 821-827.

25. Hellings N, Stinissen $P$ (2008) Tovaxin, radiation-attenuated, patient-specific T-cells for the therapeutic vaccination of multiple sclerosis. Curr Opin Investig Drugs 9: $534-540$.

26. Spohn G, Guler R, Johansen P, Keller I, Jacobs M, et al. (2007) A virus-like particle-based vaccine selectively targeting soluble TNF-alpha protects from arthritis without inducing reactivation of latent tuberculosis. J Immunol 178: 7450-7457.

27. Ludvigsson J (2010) GAD-alum (Diamyd)--a new concept for preservation of residual insulin secretion. Expert Opin Biol Ther 10: 787-799.

28. Hinke SA (2008) Diamyd, an alum-formulated recombinant human GAD65 for the prevention of autoimmune diabetes. Curr Opin Mol Ther 10: 516-525.

29. (2011) DIAPREV-IT study of diabetes therapy Diamyd® receives further funding and will continue despite disappointing Phase III trial results. Immunotherapy 3: 923-924.

30. Lernmark A, Larsson HE (2013) Immune therapy in type 1 diabetes mellitus. Nat Rev Endocrinol 9: 92-103.

31. Faustman DL, Wang L, Okubo Y, Burger D, Ban L, et al. (2012) Proof-ofconcept, randomized, controlled clinical trial of Bacillus-Calmette-Guerin for treatment of long-term type 1 diabetes. PLoS One 7: e41756.

32. Silva DG, Charlton B, Cowden W, Petrovsky N (2003) Prevention of autoimmune diabetes through immunostimulation with $Q$ fever complement-fixing antigen. Ann N Y Acad Sci 1005: 423-430.

33. Hoogsteder PH, Kotz D, van Spiegel PI, Viechtbauer W, Brauer R, et al. (2012)
The efficacy and safety of a nicotine conjugate vaccine (NicVAX ${ }^{\circledR}$ ) or placebo co-administered with varenicline (Champix $\left.{ }^{\circledR}\right)$ for smoking cessation: study protocol of a phase Ilb, double blind, randomized, placebo controlled trial. BMC Public Health 12: 1052.

34. Heading CE (2003) NicVAX (Nabi Biopharmaceuticals). IDrugs 6: 1178-1181.

35. Novartis (2009) Study to Evaluate the Efficacy, Safety, Tolerability and Immunogenicity of $100 \mu \mathrm{g} \mathrm{NIC002} \mathrm{Vaccine} \mathrm{in} \mathrm{Cigarette} \mathrm{Smokers} \mathrm{Who} \mathrm{Are}$ Motivated to Quit Smoking.

36. Maurer P, Jennings GT, Willers J, Rohner F, Lindman Y, et al. (2005) A therapeutic vaccine for nicotine dependence: preclinical efficacy, and Phase I safety and immunogenicity. Eur J Immunol 35: 2031-2040.

37. Heading CE (2002) TA-CD. Xenova. IDrugs 5: 1070-1074.

38. Petrovsky N, Aguilar JC (2004) Vaccine adjuvants: current state and future trends. Immunol Cell Biol 82: 488-496.

39. Nikolai Petrovsky, Susanne Heinzel, Yoshikazu Honda, A. Bruce Lyons (2007) New-Age Vaccine Adjuvants: Friend or Foe? Biopharm International 20: 24-33.

40. Cooper PD, Petrovsky N (2011) Delta inulin: a novel, immunologically active stable packing structure comprising $\hat{i}^{2}-D-[2->1]$ poly(fructo-furanosyl) $\hat{I} \pm-D-$ glucose polymers. Glycobiology 21: 595-606.

41. Petrovsky N, Cooper PD (2011) Carbohydrate-based immune adjuvants. Expert Rev Vaccines 10: 523-537.

42. Honda-Okubo Y, Saade F, Petrovsky N (2012) Advax ${ }^{\top M}$, a polysaccharide adjuvant derived from delta inulin, provides improved influenza vaccine protection through broad-based enhancement of adaptive immune responses. Vaccine 30: 5373-5381.

43. Saade F, Honda-Okubo Y, Trec S, Petrovsky N (2013) A novel hepatitis $B$ vaccine containing Advaxâ, $\phi$, a polysaccharide adjuvant derived from delta inulin, induces robust humoral and cellular immunity with minimal reactogenicity in preclinical testing. Vaccine 31: 1999-2007.

44. Petrovsky N (2008) Freeing vaccine adjuvants from dangerous immunological dogma. Expert Rev Vaccines 7: 7-10. 